\title{
Dynamic shear fracture of an explosively-driven metal cylindrical
} shell

\author{
Guowu Ren* and Zhaoliang Guo, Cheng Fan, Tiegang Tang, Haibo Hu \\ Institute of Fluid Physics, Chinese Academy of \\ Engineering Physics, Mianyang 621999, China ${ }^{\dagger}$
}

(Dated: March 15, 2016)

\begin{abstract}
This work investigates shear fracture behavior occurring in a titanium alloy cylinder internally filled with high explosives through the use of photonic Doppler velocimetry(PDV) array, high-speed framing camera and soft capture tank. The real-time velocity profiles diagnosed by PDV array display from overlapping to scattering, corresponding to the cylinder from uniform expansion to onset of fracture. In addition to the general findings obtained from individual diagnostics, combined analysis from the experimental measurements determines sliding velocity between sheared cracks and the overall adiabatic shear failure process of the metal cylinder is discussed.
\end{abstract}

PACS numbers: Valid PACS appear here

Keywords: cylindrical shell; velocity profile; fracture

* guowu.ren@yahoo.com

$\dagger$ Telephone number: +86-816-2493325 


\section{INTRODUCTION}

Dynamic response of metal cylindrical shells at high strain rates receives considerable attention in the industrial and military applications, which includes expanding deformation, crack initiation and propagation, and final rupture. Physical mechanisms of crack formation, fracture criteria and fragmentation behavior are most focused on by experimental measurement, model development and numerical simulation. Earlier in 1943, Gurney[1] had proposed an empirical expression to estimate the ultimate velocity of fragments generated from the metal cylinder internally filled with high explosives. Taylor[2] suggested a theory about failure process of the cylinder related to stress states; however, it is only applicable to radial fracture. Hoggatt and Recht[3] further extended to shear fracture mode by perfecting Taylor's model. Mott[4] and Grady[5, 6] separately investigated dynamic fragmentation behavior from statistics-based and energy-based perspectives, predicting the relationship between fragment number and loading strain rates and building a systematic theoretical model for fragmentation, which lays the foundation for conducting numerical simulations[7-11].

Compared to the comprehensive understanding of fragment formation and its statistic distribution, quantitative investigation on dynamic process for the metal cylindrical shell is still sparse, particularly for dominated shear fracture, which originates from adiabatic shear localization. Up to date, available experimental methods [12-20] for characterizing shear fracture failure mainly have: 1) high-speed framing camera, which determines the fracture mode from the photographed images of shell surface; 2) metallurgical characterization, which observes micrograph of the recovered fragments, e.g. large strain localization represents adiabatic shear band. However, these findings only provide qualitative information on the shear fracture for the rupture of the metal cylinder, which is insufficient to unravel the failure mechanism, build shear fracture model and perform numerical simulations. Therefore, it is of considerable importance to gain some quantitative data on shear fracture by improving the diagnostic technique and analytical capability.

In this paper, a combination of PDV array, high-speed framing camera and soft-recovery technique is utilized to investigate dynamic shear fracture response of the titanium alloy(Ti6Al4V) cylindrical shell internally filled with high explosives. The photographed frames offer the circumferential strain of crack initiation and rupture. The collected fragments are used for obtaining the average fragment length and metallurgical examination. 
Moreover, comparative analysis from real-time results obtains the sliding velocity between sheared cracks as well as elucidates failure character of the metal cylinder.

\section{EXPERIMENTAL LAYOUT AND DIAGNOSTICS}

The whole fielding layout is illustrated in Fig. 1, including experimental setup and diagnostics. The former is composed of a metal cylinder, a high explosive and a detonator, and the latter consists of high-speed framing camera, PDV array and capture tank. The metal cylindrical shell is machined from Ti6Al4V alloy, which has a mass density of $\rho=4.43$ $\mathrm{g} / \mathrm{cm}^{3}$ and is prone to adiabatic shear band formation. The metal cylinder has an outer radius of $52 \mathrm{~mm}$, a wall thickness of $6 \mathrm{~mm}$ and a length of $140 \mathrm{~mm}$. The filled high explosives are JOB-9003 (87 wt.\%HMX crystals)[21], which geometry has a diameter of $40 \mathrm{~mm}$ and a length of $120 \mathrm{~mm}$. The JOB-9003 has a mass density of $\rho=1.849 \mathrm{~g} / \mathrm{cm}^{3}$, a detonation velocity of $\mathrm{D}=8.7 \mathrm{~km} / \mathrm{s}$ and a Chapman - Jouguet(CJ) pressure of $P_{C J}=35.2 \mathrm{GPa}$.

The high-speed framing camera is used for recording 40 images of the metal cylinder surface at a rate of 2 frames per $\mu$ s. To avoid high-speed metal fragments directly impacting the camera, a reflecting mirror is oriented at an angle of $45^{\circ}$ with respect to the axis of metal cylinder. An argon gas in a large plastic bag as a illuminator, is explosively shocked to frontally light the cylinder with the background of a white gauze. To clearly photograph the cylinder surface, two illuminators are placed separately along the $\pm 45^{\circ}$ direction. The capture tank filled with watered sawdust is positioned below the experimental setting at a distance of about $200 \mathrm{~mm}$ and only collects a fraction of metal fragments. Since we do not take into account the statistical distribution of fragments, these recovered fragments are adequate for metallurgical examination and length measurement. Scanning electron microscopy (SEM) is used for characterizing the fracture surface and microstructure for the metal fragments.

PDV technique is able to accurately measure high velocity profile of a moving body and has been widely employed for explosively loaded metal cylinders; however, to date it only restricts to individual measured point. Actually, theoretical formula concerned about the relationship between average fragment length and loading strain rate derived by Grady[5] could be appropriate for individual fragment, implying the dependence of fragment spacing on the fragment velocity, as will be illustrated in great detail in Section III. B. Therefore, we 
contrive a PDV array arranged on a local position of shell surface along the circumferential direction, which ensures PDV probes to span multiple microscopic cracks. According to previous experimental results about average fragment width and PDV measured depth for Ti6Al4V alloy, designed PDV array is depicted in Fig. 2(a), which is an arc stand with a radius of $76 \mathrm{~mm}$. This arc, made from aluminium alloy, includes six PDV probes normal to the shell surface and angle between probes is $3^{\circ}$. In the case of probe layout, distance between them on the shell surface is $1.36 \mathrm{~mm}$, which is larger than the focal spot, preventing the probe from overlapping of light route. Figure 2(b) illustrates the practical situation of PDV array placed in the middle of the metal cylinder.

\section{EXPERIMENTAL RESULTS}

\section{A. Framing images}

As a traditional approach for diagnosing dynamic response of the metal cylinder, highspeed framing camera photographs multiple images of shell surface at short time and enables us to obtain a visible observation, such as necking, crack initiation or detonation product leakage. Estimate from these images roughly acquires the circumferential strain of crack initiation and complete rupture of cylinder at different moments. Figures 3(a) and (b) separately display the framing pictures of $\mathrm{t}=17.0 \mu \mathrm{s}$ and $\mathrm{t}=22.5 \mu \mathrm{s}$. By defining the circumferential strain $\epsilon_{c}=\left(D-D_{0}\right) / D_{0}$, where $D_{0}$ is an initial diameter and $D$ is a deformed diameter. Calculated from these two images, $\epsilon_{c}=0.19$ denotes crack initiation and $\epsilon_{c}=1.00$ is for detonation product leakage. In light of circumferential strain at the same location of the cylinder, such as, $\epsilon_{c}=0.40$ at $\mathrm{t}=17.0 \mu \mathrm{s}$ in Fig. 3(a) and $\epsilon_{c}=0.77$ at $\mathrm{t}=22.5 \mu \mathrm{s}$ in Fig. $3(\mathrm{~b})$, average expanding velocity is evaluated to be $1.90 \mathrm{~km} / \mathrm{s}$, close to the limiting Gurney velocity $1.99 \mathrm{~km} / \mathrm{s}$. The exact ultimate velocity measured by PDV is $1.85 \mathrm{~km} / \mathrm{s}$, as seen in next section. Observed from surface morphology of the cylinder shell, it can be determined that fracture characteristic is shear mode and short crack along the longitudinal direction, completely differing from the radial fracture proposed by Taylor[2].

It is also noted from Fig. 3(b) that bright region represents the sliding crack surface and the dark is for crack surface. Compared to Fig. 3(a), the former becomes wider but the latter keeps nearly unchanged; therefore, we are able to evaluate the relatively sliding 
velocity between shear cracks, and fragment width along the transverse direction. For the sake of conveniently calculating these values, Figs. 3(a) and (b) are inverted into Figs. 3(c) and $(d)$, respectively, where white is fragment surface and dark is sliding crack surface. To improve the accuracy on estimate of dark width, a marked site approximatively located on the opposing surface of PDV measurement is chosen and close to middle zone of the framing image. Calculation of dark width from the marker obtains a value of 4 pixels. By referencing a width of $12 \mathrm{~mm}$ (58 pixels) occupied by static PDV stand, sliding width between Fig. 3(c) and Fig. 3(d) is on the order of $0.83 \mathrm{~mm}$. In terms of a time difference of $5.5 \mu$ s, relatively sliding velocity along the transverse direction is approximated to be $150 \mathrm{~m} / \mathrm{s}$. Adopting the measured approach above, average width of white region marked as fragment is about 14 pixels, corresponding to $2.89 \mathrm{~mm}$, which is in good agreement with the fragment width averaged from the recovered fragments as listed in Section III. C.

\section{B. Velocity profiles of PDV array}

The measured real-time velocity profiles using PDV array are shown in Fig. 4(a), where points a-f are one-to-one correspondence to the marked positions in Fig. 2(a). Before third maximum value of velocity profile, six plots are exactly overlapped, indicating the uniformly expanding deformation of the measured region. The rise times of velocity profiles are separately $11.10 \mu \mathrm{s}, 11.00 \mu \mathrm{s}, 11.02 \mu \mathrm{s}, 11.09 \mu \mathrm{s}, 11.01 \mu \mathrm{s}$ and $11.06 \mu \mathrm{s}$ from point a to point $\mathrm{f}$, and the corresponding maximum velocities are $1.158 \mathrm{~km} / \mathrm{s}, 1.193 \mathrm{~km} / \mathrm{s}, 1.183 \mathrm{~km} / \mathrm{s}$, $1.165 \mathrm{~km} / \mathrm{s}, 1.173 \mathrm{~km} / \mathrm{s}$ and $1.188 \mathrm{~km} / \mathrm{s}$. The maximum error of rise time is $0.1 \mu \mathrm{s}$, implying an assembled error of $\sim 1.0 \mathrm{~mm}$ among the PDV probes along the axial direction. Averaging

the velocity at the rise time obtains the initial loading strain rate of $4.5 \times 10^{4} / \mathrm{s}$. Further calculation based on the time interval of shockwave reverberating through the wall thickness gains a shock wave speed of $5.91 \mathrm{~km} / \mathrm{s}$.

Seen from Fig. 4(a), it can be noted that at about $\mathrm{t}=15.0 \mu \mathrm{s}$ six velocity curves begin to disperse. Simply integrating the velocity plot obtains the circumferential strain $\epsilon_{c}=0.19$, corresponding to the framing image as shown in Fig. 4(b). It is apparent that observed from the cylinder surface in Fig. 4(b), crack emerges in this region measured by PDV array, illustrating that branching of velocity profiles reveals crack initiation. Thus, duration from expanding deformation to crack initiation lasts for $4.0 \mu \mathrm{s}$ and to and fro behavior of 
shockwave disappears upon crack initiation releasing the Mott wave. Note that velocity history of point e reveals third reverberation after $\mathrm{t}=15.0 \mu \mathrm{s}$, indicating the sustaining circumferential stress imposing on this sites and also implying that the fragment probed by PDV is much wider than the others.

Meanwhile, it can be further seen that the PDV measured time of point c reaches 37.0 $\mu$ s against the maximum time of $31.5 \mu$ s pictured by camera shown in Fig. 4(c). Observed from this image, the leaking detonation products have emerged at the locations nearby the PDV stand while not affecting the PDV measurement of point c. In total, velocity profiles of Fig. 4(a) do not reveal an evident jump, meaning that PDV probes do not span transverse or longitudinal crack surface (PDV is fixed.) and measure the different fragments, while the maximum measured time is influenced by appearance of denotation product.

In the following, we are devoted to the analysis of discrete velocity plots in the inset of Fig. 4(a), where time ranges from $15.0 \mu \mathrm{s}$ to $30.0 \mu \mathrm{s}$. The previous analysis has indicated the velocity branching stemming from crack initiation of cylinder surface. According to the energy-based conservation rule for fragment formation, where kinetic energy equals fracture energy at high strain rate $\left(>10^{4} / \mathrm{s}\right)$, average fragment length $l_{f}$ could be written as

$$
l_{f}=\left(\frac{24 \Gamma}{\rho \dot{\epsilon}_{0}^{2}}\right)^{1 / 3}
$$

where $\Gamma$ is fracture energy per unit area, $\rho$ is mass density and $\epsilon_{0}$ is loading strain rate. Although the characteristic length is a generalized formalism for the expanding ring or shell experiment, in fact it is appropriate for describing the energy relationship for individual fragment. The shear fracture energy used here has the expression,

$$
\Gamma=\frac{\rho c}{\alpha}\left(\frac{9 \rho^{3} c^{2} \chi^{3}}{Y^{3} \alpha^{2} \epsilon_{0}}\right)^{1 / 4}
$$

where $c$ is specific heat, $\chi$ is thermal diffusion coefficient, $\alpha$ is thermal softening coefficient and Y is plastic flow stress. Combination with Eqs.(1) and (2) and employing the loading strain rate defined as velocity divided by radius yield,

$$
V \propto l_{f}^{-4 / 3}
$$

${ }_{160}$ Thus, larger velocity in the inset of Fig. 4(a) represents narrower fragment spacing. 


\section{Analysis of recovered fragments}

In the capture tank, a fraction of metal fragments are recovered and cleaned. A detailed inspection of all these fragments indicates shear fracture mode, shown in Fig. 5(a). By choosing several well-defined fragments with both inner and outer surfaces, we make the measurement of fragment width, length of crack surface and angle with respect to the original inner surface in the cross-sectioned area, which are separately averaged on the order of $\sim$ $2.7 \mathrm{~mm}, \sim 8.5 \mathrm{~mm}$ and $\sim 35^{\circ}$. Note that this angle is lower than that of around $45^{\circ}$ for shear fracture as proposed in the literatures $[3,16]$. It is estimated from these measured data that fragment thickness is roughly $5.8 \mathrm{~mm}$, indicating a small deformation along the radial direction, in contrast to an initial thickness of $6 \mathrm{~mm}$.

A careful examination of fragment in Fig. 5(a) finds that there exists an evident step close to the outer surface on the crack surface. Based on metallurgical characterization at the two sides of this step, denoted by A and B in Fig. 5(a), the SEM images are displayed in Fig. 5(b) and (c), respectively. Figure 5(b) exhibits numerous microvoids which represents the ductile fracture, while figure 5(c) is completely distinguishable from Fig. 5(b), revealing a morphology like flow. The latter is attributed to local adiabatic shear formation generated from the inner surface, displayed in Fig. 6. However, further observation reveals a shear crack opening in the middle of the fragment.

\section{DISCUSSION}

Based on the photographed images by high-speed camera, sliding velocity between sheared crack surface along the transverse direction, denoted by $V_{\text {image }}$, is estimated to be $150 \mathrm{~m} / \mathrm{s}$. The schematic model about the cross section of the metal cylinder is shown in Fig. 7. To facilitate the analysis of the sliding process, in Fig. 7 we define the photographed direction as $\mathrm{y}$ axis and transverse direction as $\mathrm{x}$ axis. Other definitions are listed, where site A corresponds to site 1 in Fig. $3, l$ is the length of crack surface, $\alpha$ is angle between inner surface and crack surface, equal to $35^{\circ}$ and $V_{\text {sliding }}$ is sliding velocity between sheared cracks. In terms of the geometrical relationship, the angle $\beta$ is calculated to be $34.64^{\circ}$. Therefore, the relative sliding velocity $V_{\text {sliding }}$, expressed as $V_{\text {image }} / \cos (\beta)$, is $182 \mathrm{~m} / \mathrm{s}$. Using $V_{\text {sliding }}$, the sliding velocity along the radial direction, denoted as $\mathrm{OB}$, is $140 \mathrm{~m} / \mathrm{s}$. Assuming 
the uniformity of relative velocity between the sheared fragments, this velocity along OB direction consistent with PDV probed direction, compares well with the maximum relative velocity of about $100 \mathrm{~m} / \mathrm{s}$ determined from the inset of Fig. 4(a).

Combination with high-speed framing images and fragment characterization could determine the shear failure process of titanium alloy cylinder subjected to the high strain-rate loading. When the explosive shockwave drives the metal cylinder, inner surface sharply enters into the circumferential compressive state and initiates the numerous adiabatic shear bands due to inner surface heterogeneity. Once shockwave reaches the outer surface, the cylinder rapidly expands outwards along the radial direction and the outer region begins to undergo the circumferential tensile stress. Afterwards, the release wave from outer wall reflect back inner region, inducing the decreasing velocity, shown in the velocity profiles of Fig. 4(a). Since the circumferential inner zone is located at the compressive stress imposed by detonation pressure, adiabatic shear bands grow along plane of maximum shear stress while the angle is only $35^{\circ}$ measured from the recovered fragment. Meanwhile, the metal cylinder keeps on radial expansion, which leads to the circumferential stress to increase until shear crack on the outer surface initiates. The inner region keeps the sliding movement owing to the large adiabatic localization, but there have been some voids appeared within the adiabatic shear band, seen from Fig. 6. As voids coalesce, crack in the inner region forms and move outwards with a sliding velocity of $180 \mathrm{~m} / \mathrm{s}$. When the adiabatic shear crack from inner region intersects with one from outer region, achieving the complete opening, the denotation products steam through the fractured surface, which causes the ultimate rupture of the metal cylinder.

\section{CONCLUSION}

In this paper, we experimentally conduct the quantitative measurements about shear fracture of the Ti6Al4V cylindrical shell undergoing the high explosive loading. High-speed framing camera gives the circumferential strain relevant to crack initiation, detonation product leakage and assess the failure mode from the observation of the cylinder surface. The firstly-used PDV array measures the real-time velocity profiles within the circumferential region, capturing the distinct property that velocity transitions from uniform to scattering corresponding to deformation followed by crack initiation. From the velocity profile, initial 
loading strain rate, shockwave speed and ultimate velocity of fragment could be accurately acquired. We also make the measurement of the recovered fragments including the sheared angle, length of crack surface and fragment width and characterize the microscopic structure. In addition to these above results, combined analysis from the diagnostic findings provides a clear understanding about the failure process and estimates the sliding velocity between shear cracks. Totally, these experimental results provide fruitful information on shear fracture of the explosively-loaded metal cylinder, which contributes to the understanding of this dynamic response and development of sliding physical model. However, PDV array and high-speed framing camera cannot diagnose the same location, which deserves the ongoing exploration in the future.

\section{ACKNOWLEDGMENTS}

We would like to thank S. W. Zhang for valuable discussions, H. M. Su for setup design, S. J. Wen, S. Jin and J. Liu for experimental measurement. This work is sponsored by the National Science Foundation of China(No. 11172279), which is gratefully acknowledged.

[1] Gurney RW. The initial velocity of fragments from bombs, shells and grenades, Army Ballistic Research Laboratory Report No. 405,1943.

[2] Taylor GI. Analysis of the explosion of a long cylindrical bomb detonated at one end. In: Scientific Papers of G. I. Taylor, vol. III, Cambridge: Cambridge University Press: 1963. No. 30 , pp. $277-286$

[3] Hoggatt CR, Recht RF. Fracture behavior of tubular bombs. J Appl Phys 1968; 39(3): 186562.

[4] Mott NF. Fragmentation of shell cases. Proc R Soc London, Ser A 1947; 189: 300-8.

[5] Grady DE. Fragmentation of Rings and Shells: The Legacy of N. F. Mott. New York: Springer; 2006.

[6] Grady DE, Olsen ML. A Statistics and Energy Based Theory of Dynamic Fragmentation. Int J Impact Eng 2003; 29: 293-306. 
[7] Miller O, Freund LB, Needleman A. Modeling and simulation of dynamic fragmentation in brittle materials. Int J Fract 1999; 96: 101-125.

[8] Pandolfi A, Krysl P, Ortiz M. Finite element simulation of ring expansion and fragmentation: the capturing of length and time scales through cohesive models of fracture. Int J Fract 1999; 95: $279-297$

[9] Kong XS, Wu WG, Li J, Liu F, Chen P, Li Y. A numerical investigation on explosive fragmentation of metal casing using Smoothed Particle Hydrodynamic method. Mater Des 2013; 51: 729-741.

[10] Mariotti C, Michaut V, Molinari JF. Modeling of the fragmentation by Discrete Element Method. DYMAT 2009; 2009: 1523-1528.

[11] Goloveshkin VA, Myagkov NN. Fragmentation model for expanding cylinder. Int J Fract 2014; 187: $239-243$

[12] Mock W, Holt JrWH. Fragmentation behavior of Armo iron and HF-1 steel explosive-filled cyinders. J Appl Phys 1983; 54: 2344-2351.

[13] Martineau RL, Anderson CA, Smith FW. Expansion of Cylindrical Shells Subjected to Internal Explosive Detonations. Exp Mech 2000; 40: 219-225.

[14] Singh M, Suneja HR, Bola MS, Prakash S. Dynamic tensile deformation and fracture of metal cylinders at high strain rates. Int J Impact Eng 2002; 27: 939-954.

[15] Campbell GH, Archbold GC, Hurricane OA, Miller PL. Fragmentation in biaxial tension. J Appl Phys 2007; 101: 033540

[16] Goto DM, Becher R, Orzechowski TJ, Springer HK, Sunwoo AJ, Syn CK. Investigation of the fracture and fragmentation of explosively driven rings and cylinders. Int J Impact Eng 2008; 35: $1547-1556$.

[17] Hiroe T, Fujiwara K, Hata H, Takahashi H. Deformation and fragmentation behavior of exploded metal cylinders and the effects of wall materials, configuration, explosive energy and initiated locations. Int J Impact Eng 2008; 35: 1578-1586.

[18] Zhang H, Ravi-Chandar K. On the dynamics of localization and fragmentation-IV. Expansion of $\mathrm{Al}$ 6061-O tubes. Int J Fract 2010; 163: 41-65.

[19] Mercier S, Granier N, Molinari A, Llorca F, Buy F. Multiple necking during the dynamic expansion of hemispherical metallic shells, from experiments to modelling. J Mech Phys Solids 2010; 58: 955-982. 
[20] Bolis C, Counilh D, Lagrange JM, Frugier PA. Fragmentation of a Titanium Alloy Shell in Expansion: From Experiment to Simulation. Procedia Eng 2013;58: 672-677.

[21] Tian Y, Zhang WB, Wen MP. AE features of PBX JOB-9003 depending on loading rates. Key Eng Mater 2004; 270: 466-470. 


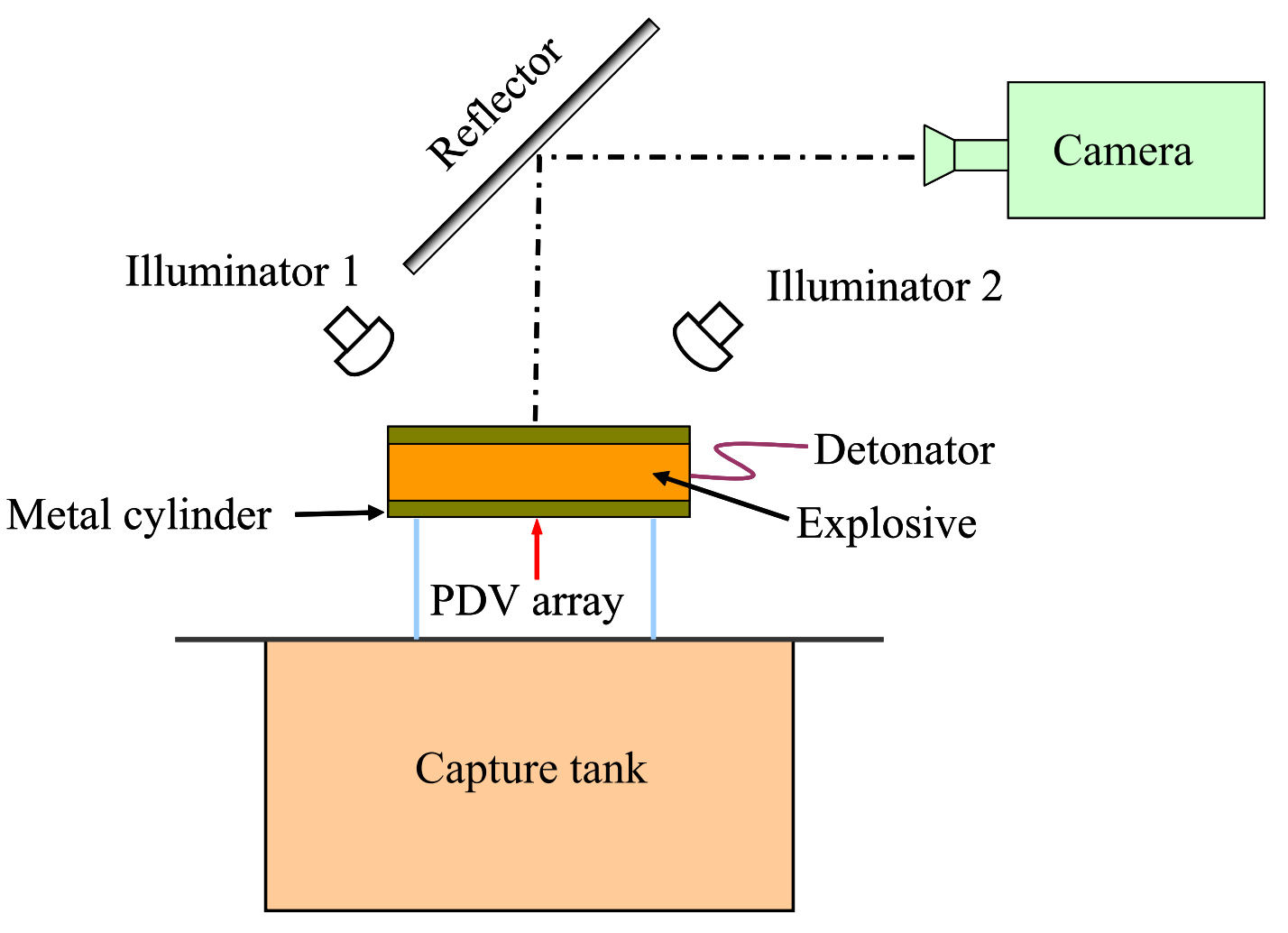

FIG. 1. Experimental layout of explosively-loaded metal cylinder and diagnostic tools.

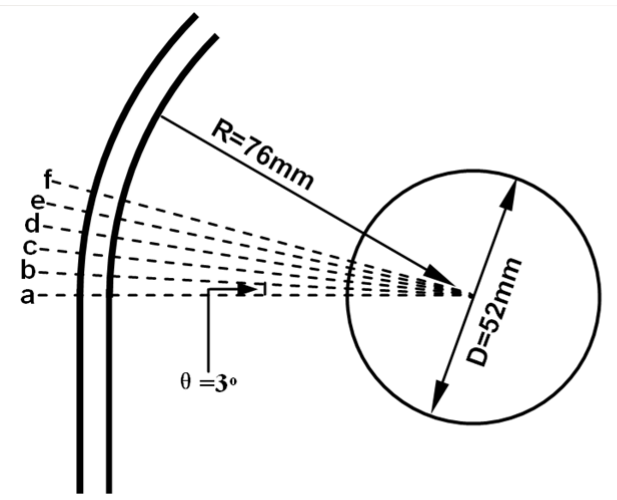

(a)

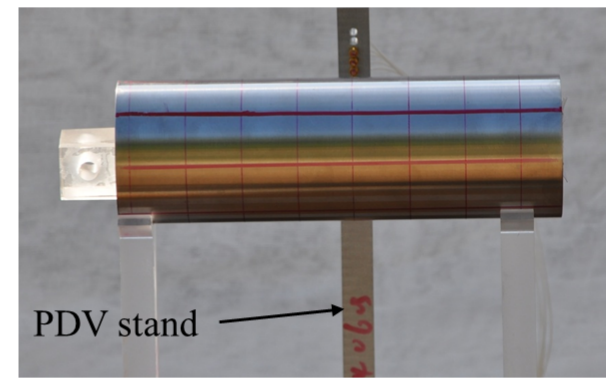

(b)

FIG. 2. (a)Schematic illustration of designed PDV array with six probes denoted by a-f. (b)Practical arrangement of PDV array on the metal cylinder surface. 

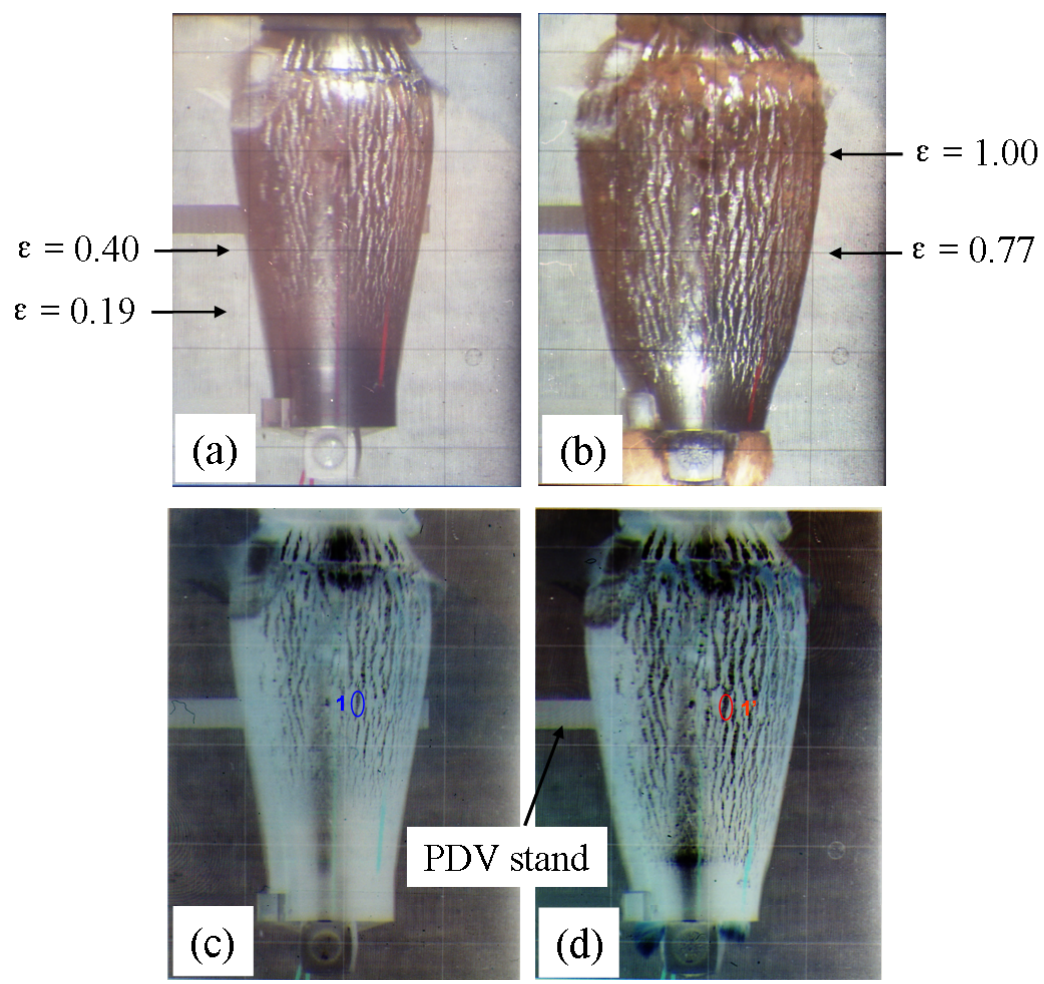

FIG. 3. Framing images including uniformly deformed expansion, crack initiation and detonation product leaking: (a)t=17 $\mu \mathrm{s}$; (b) $\mathrm{t}=22.5 \mu \mathrm{s}$; (c) a negative image of (a); (d) a negative image of (b). The sites 1 and 1' represent the fractured surface, which width increases along the sheared direction. 

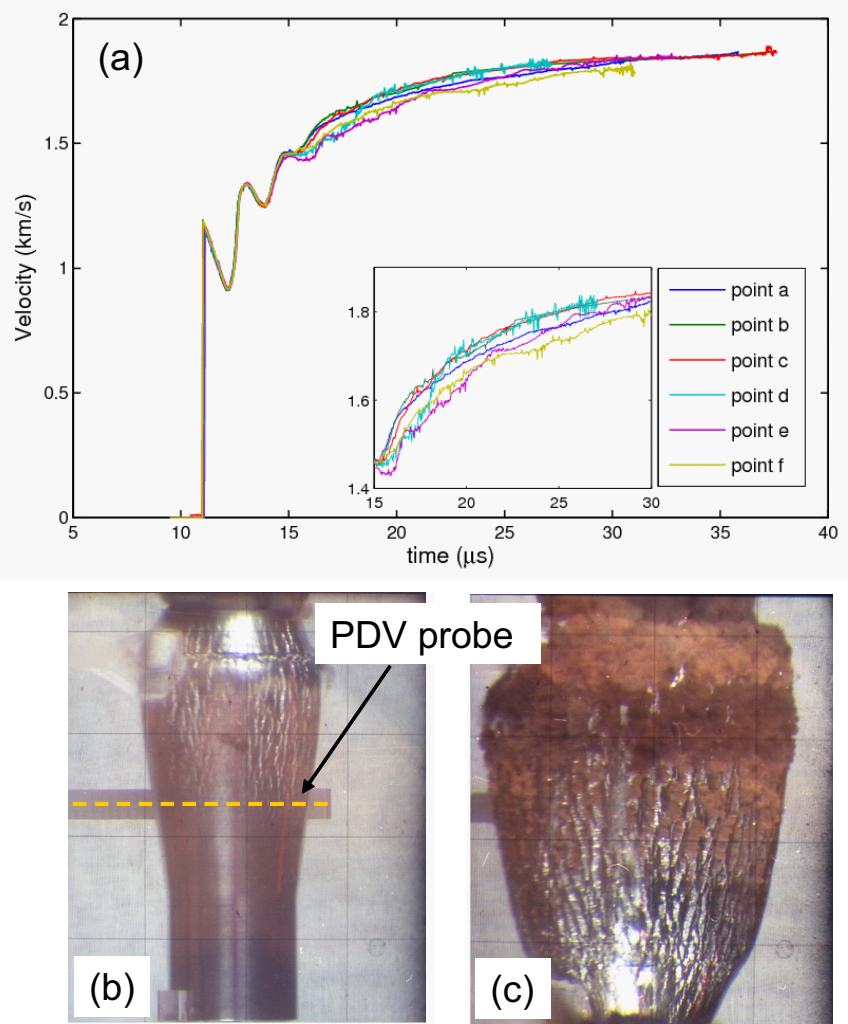

FIG. 4. (a)Velocity profiles measured by PDV array, which transitions from overlapping to scattering. (b)High-speed frame of $\mathrm{t}=15 \mu \mathrm{s}$. Crack initiation is found at the probed PDV site, corresponding to the velocity scattering in (a). (c)Last high-speed frame of $\mathrm{t}=31.5 \mu \mathrm{s}$. 

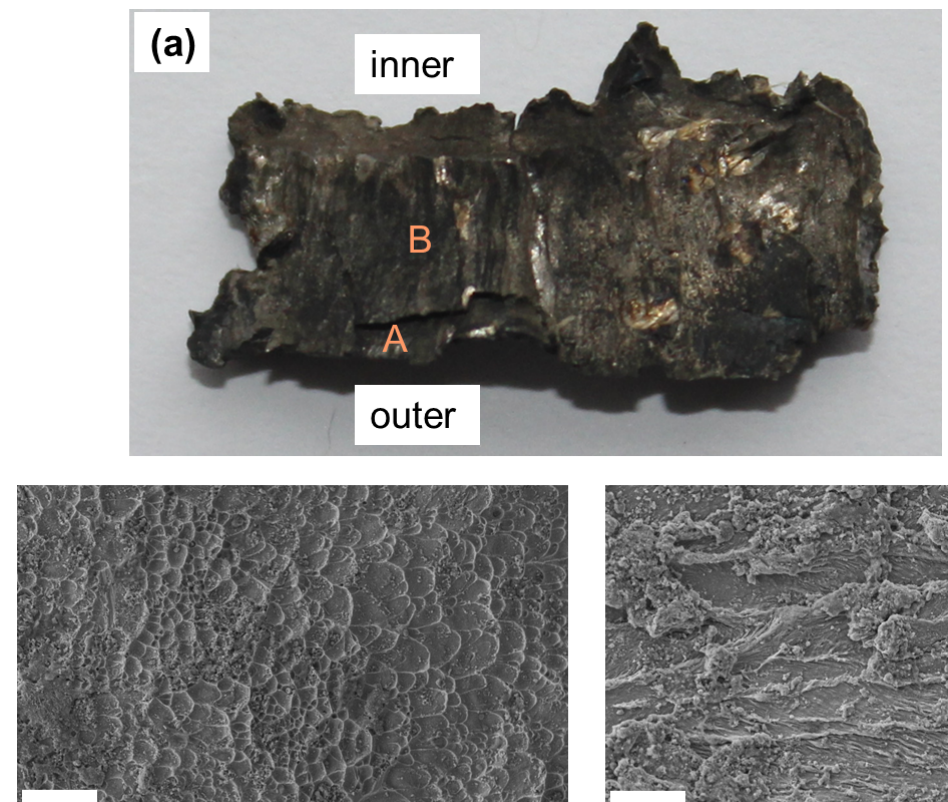

(b)

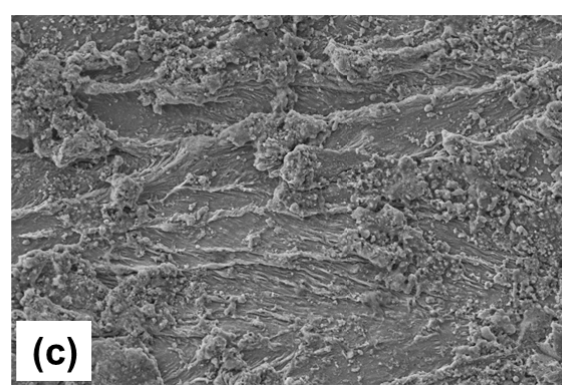

FIG. 5. (a)Typical recovered fragment dominated by shear fracture. There exists an evident step on the fractured surface nearby the outer wall. (b)Metallurgical examination of site A in (a), including numerous microvoids; (c)Metallurgical characterization of site B in (a).

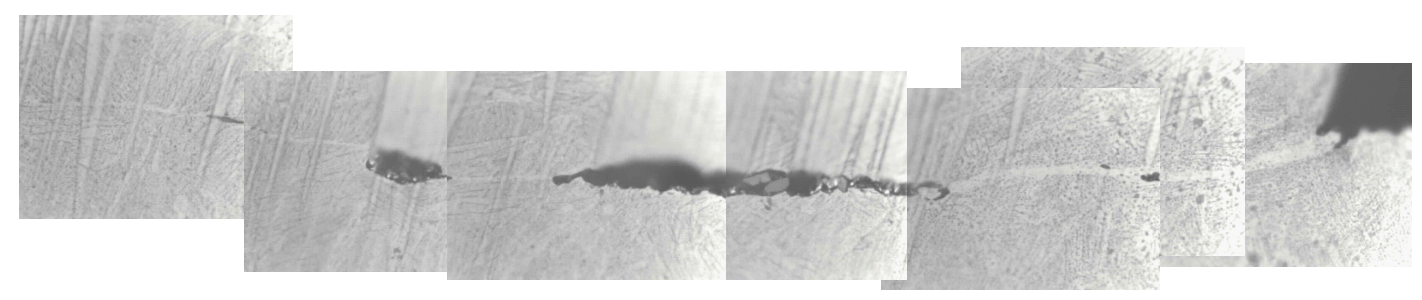

FIG. 6. Localized shear band on the cross section of the recovered fragment. It is found that crack opens in the center of the fragment. 


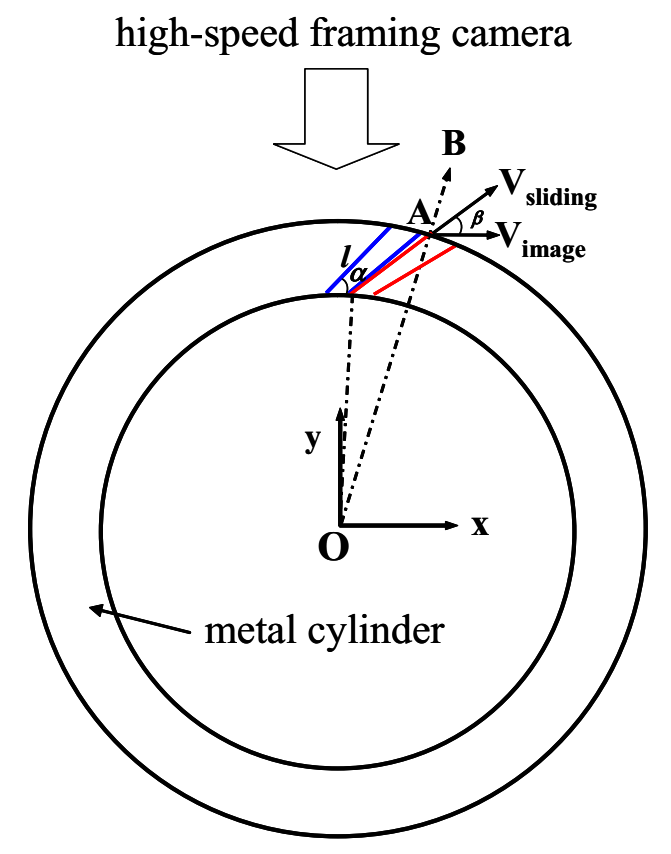

FIG. 7. Model for estimating sliding velocity between shear crack. 


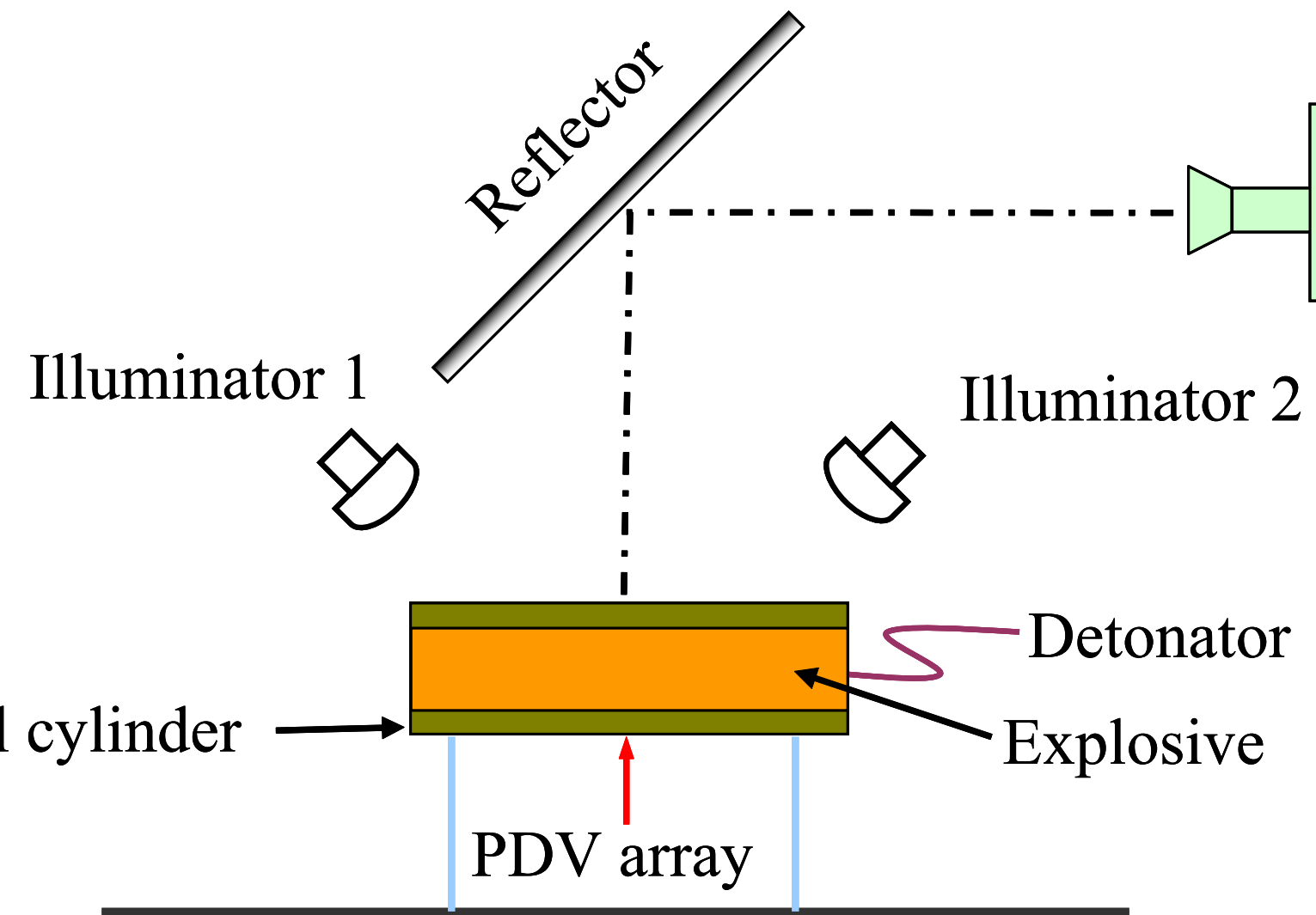

Capture tank

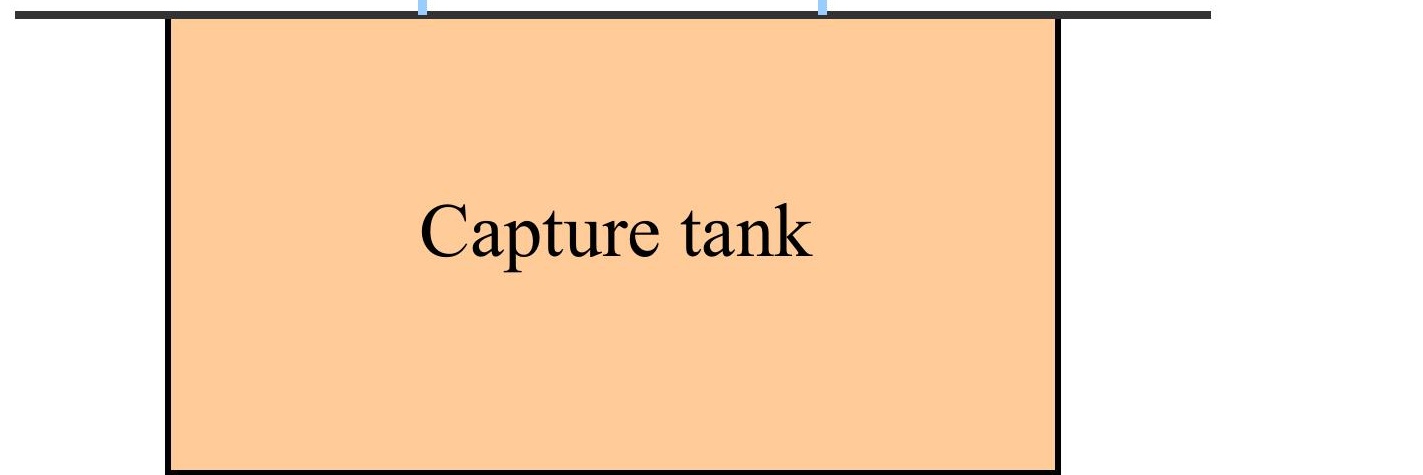



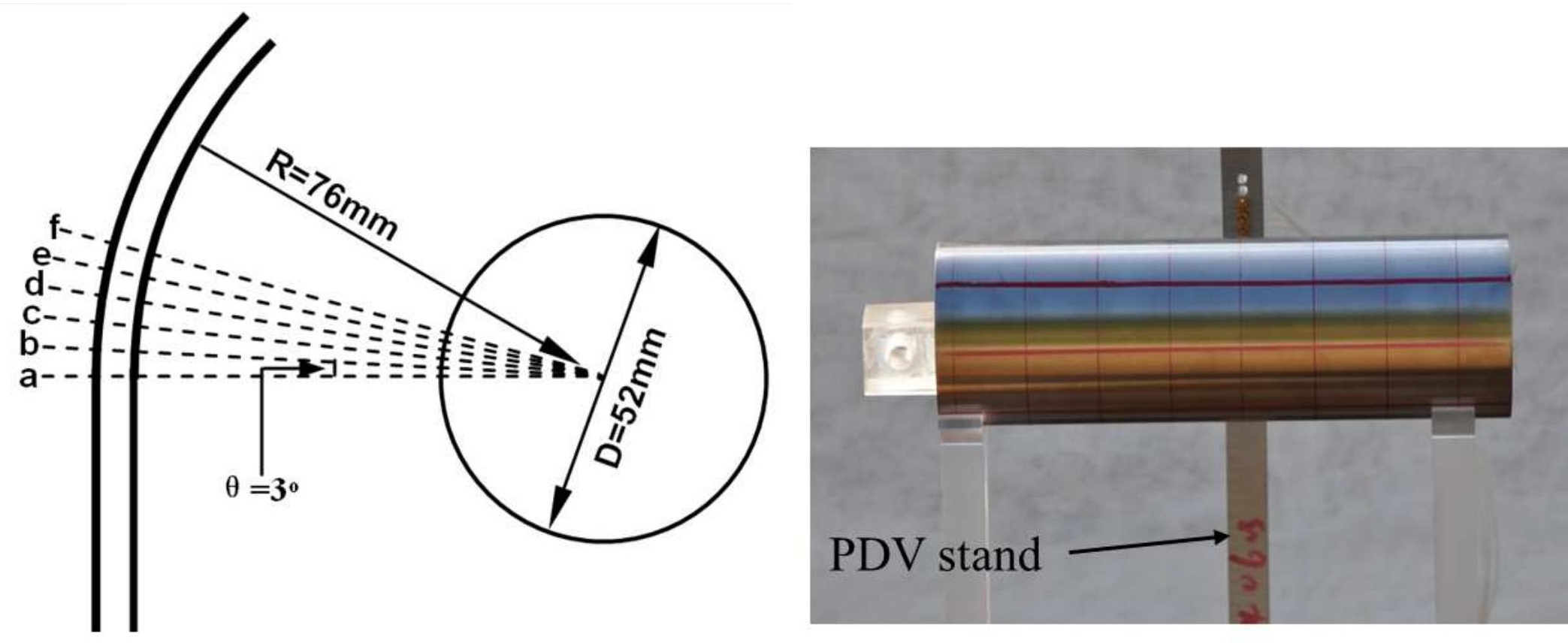

(a)

(b) 

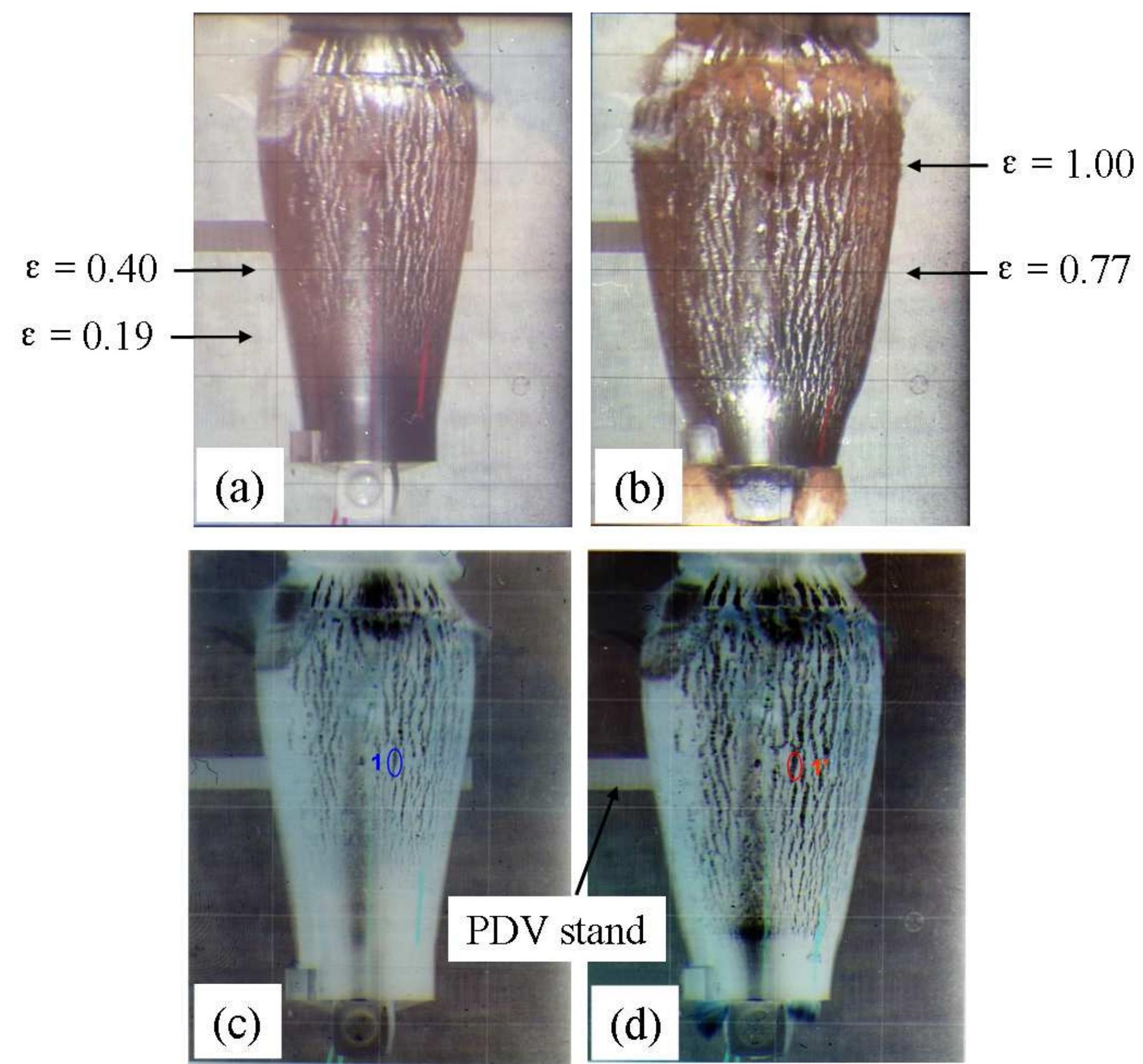

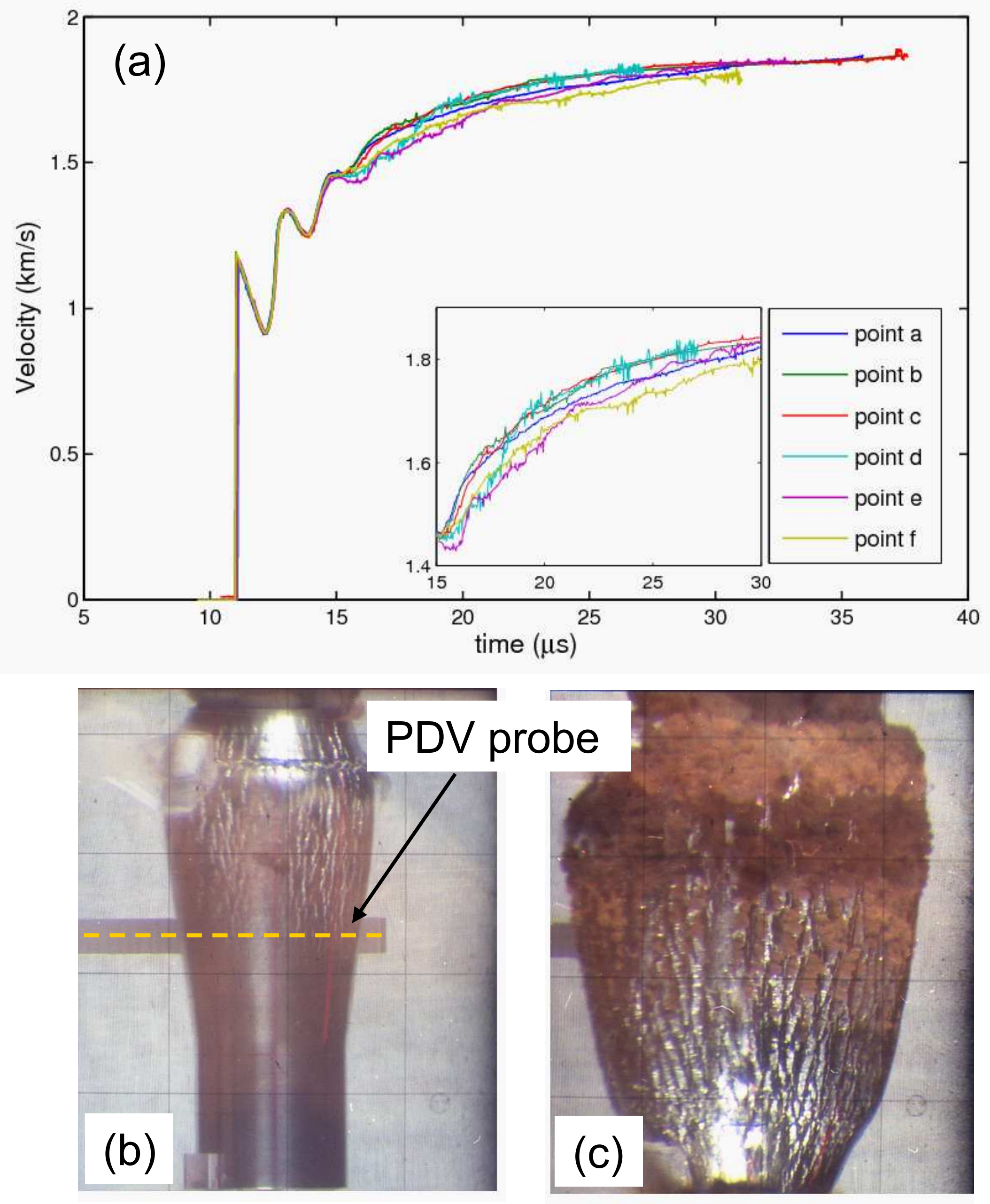

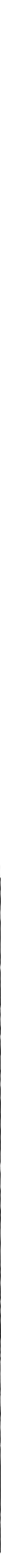


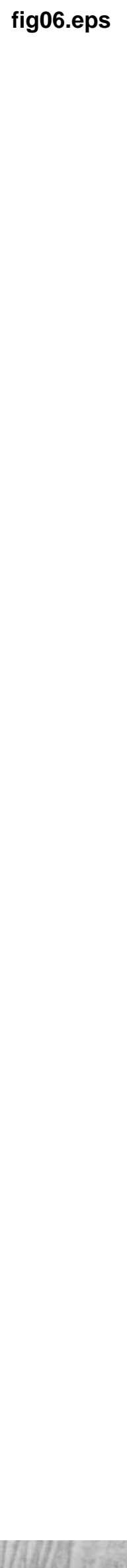

\section{s}

fiq06.eps

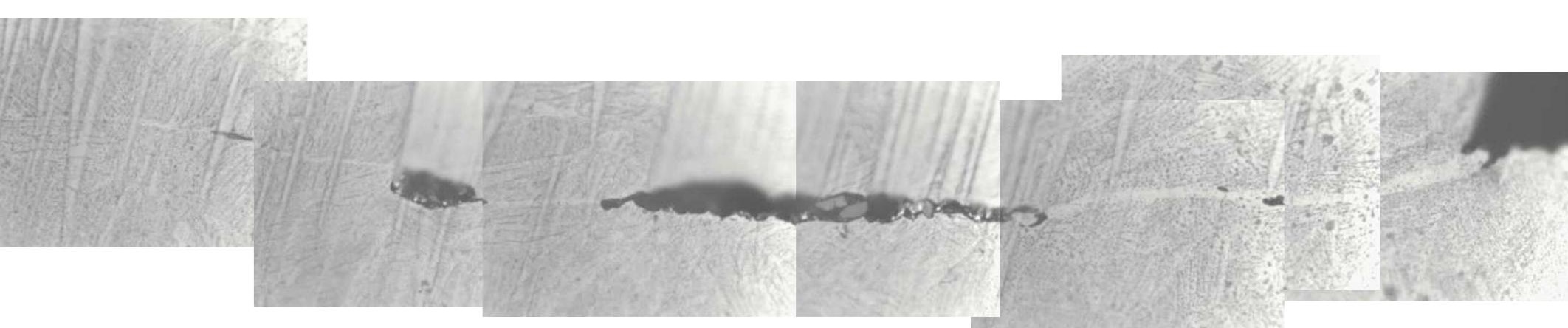


high-speed framing camera

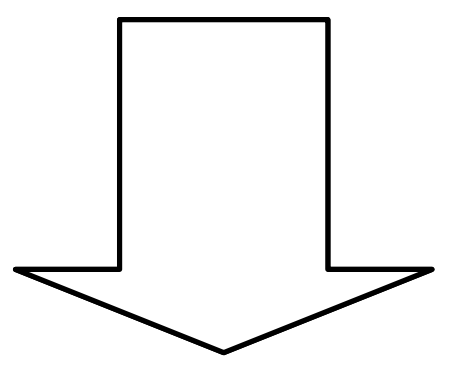

B

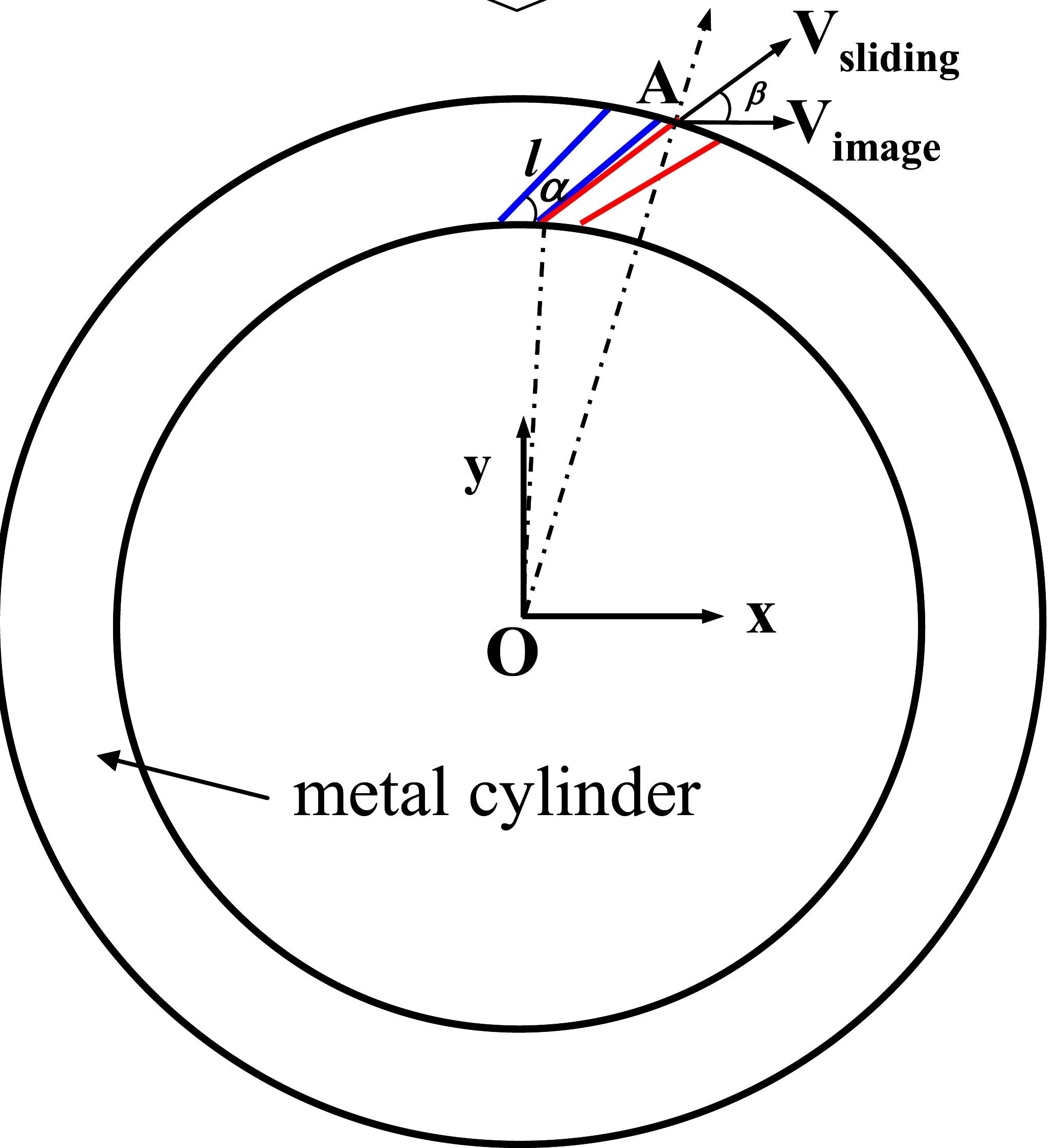

